I.S. Kostyushina ${ }^{1}$, O.V. Komarova ${ }^{1}$, A.M. Mazo ${ }^{1}$, T.V. Margieva ${ }^{1,2}$, I.V. Dvoryakovskii ${ }^{1}$, A.N. Tsygin ${ }^{1}$

Scientific Center of Children's Health, Moscow, Russian Federation

\title{
Role of central hemodynamic parameters, intimal medial thickness and endothelial dysfunction in children with renal arterial hypertension
}

\section{Author affiliation:}

Kostyushina Irina Sergeevna, pediatrician of the medical rehabilitation department for children with nephrological-urological diseases, obesity and metabolic diseases at the research institute of preventive pediatrics and medical rehabilitation of the RAMS SCCH

Address: 2, Lomonosovskiy Av., Moscow, 119991, tel.: +7 (499) 134-02-47, e-mail: ira 137@mail.ru

Article received: 18.04 .2013 , accepted for publication: 14.05 .2013

The increase in arterial hypertension is one of the most sensitive markers of the renal affection grade, including patients without chronic renal diseases; its rate is in inverse proportion to renal functions. Left ventricular hypertrophy development at chronic renal disease results from the development of increased left ventricular afterload and end diastolic pressure influence; it is a compensatory mechanism, which is closely interconnected with mortality from cardiovascular diseases. Echocardiography is the most widely used method of evaluating the condition of central hemodynamics both in adults and children with arterial hypertension. However, echocardiography does not give the researcher any complete presentation of the cardiovascular system's affection and the consequences of it in the setting of a progressing arterial hypertension. Detection of changes in the arterial wall (intimal medial thickness) and endothelial dysfunction is used to estimate the risk of developing such complications. Ultrasonic diagnostics allow to assess the wall's structure, vascular lumen and determining the intimal medial thickness. Data from a range of foreign and domestic studies allows to suggest that the level of endothelin-1 in the blood serum and its excretion with urine may reflect the renovascular damage severity. The article presents a literary review on the given issue.

Keywords: intimal medial thickness, common carotid artery, femoral artery, renal arterial hypertension, endothelin-1.

(Pediatric pharmacology. 2013; 10 (3): 32-37)

\section{INTRODUCTION}


The main death cause among patients with terminal stage of chronic kidney disease (CKD) is cardiovascular diseases $[1,2]$.

The most widely used method of evaluating condition of central hemodynamics is echocardiography both in adults and in children with arterial hypertension (AH). According to many researchers, diastolic function (DF) alterations are the earliest manifestations of intracardiac hemodynamics disorders, which precede the systolic function disorders at AH. It is possible to study systolic-diastolic dysfunctions in different $\mathrm{AH}$ progression phases using echocardiography $[3,4]$.

Diastole is the process, which provides suction effect due to the cardiac muscle's elastic recoil after systole [5]. It lasts from aortic valve closing to mitral valve closing; its duration significantly depends on preload changes, heart rate and left ventricular (LV) contractility. Novikov et al. define DF as the ability of ventricles to fill, which depends on 2 main factors: active relaxation and passive diastolic gastric properties, which in their turn depend on thickness of myocardium, degree of its fibrosis and infiltration. DF is the result of atrial and ventricular relaxation, tension and load (pressure). Myocardial DF is studied by studying transmitral and sometimes transtricuspid blood flow. Duration of the $1^{\text {st }}$ DF phase is the most important in evaluating ventricular relaxation and elasticity. It is measured from aortic valve closing to mitral valve opening and corresponds to the isovolumic relaxation time (IVRT). Transvalvular blood flow velocity increases after mitral valve opening in the setting of further ventricular pressure fall and myocardial relaxation; this corresponds to the maximal velocity of early filling (peak E) on Doppler presentations and corresponds to the III heart sound. Then, blood coasts into a ventricle. Duration of this period is known as deceleration time. After that, atrial contraction takes place (peak A), which corresponds to the IV sound beginning and late ventricular filling.

Thus, the most commonly used parameters of determining cardiac DF listed in literature are IVRT, E, A, E/A and deceleration time. All the parameters are determined on the basis of data obtained by pulse wave Doppler examination. In a healthy child, mitral blood flow velocities change with age; amplitude of peak $\mathrm{E}$ is higher than of peak $\mathrm{A}-\mathrm{E} / \mathrm{A}>1.3$.

When describing DF it is necessary to take into consideration cardiovascular system's fitness, child's initial vegetative tonus, state of the LV pre- and afterload. It has been revealed that pathologic processes of "hypertensive heart" formation in most cases result in the LV diastolic dysfunction. In case $\mathrm{AH}$ progresses and remains over a long period of time, the LV filling pressure increases together with dilatation of its cavity; this indicates "restrictive" dysfunction formation; in its turn, this points to the left ventricular systolic failure. M.C. 
Johnson et al. revealed DF alterations in the form of significant atrial peak blood flow velocity increase, E/A reduction and early diastolic filling time deceleration among 21 children with AH [6]. J.N. Chapman et al. revealed atrial filling velocity in 55 children, who had not undergone treatment concerning $\mathrm{AH}$. The authors also studied parameters of intraventricular flows of early diastolic filling on the basis of peak E parameters at the level of mitral valve leaflets and of maximal intraventricular blood flow velocity. On the basis of the obtained data, they concluded that E/A coefficient is more reliable when evaluating DF in children with $\mathrm{AH}$ [7]. A. Zurowska et al. used DF parameters to specify hypertensive syndrome character and prognosis in children with chronic renal failure [8].

It is necessary to study systolic cardiac function to obtain the complete evaluation of risk of cardiovascular complications at renal $\mathrm{AH}$.

Neurohumoral mediators - angiotensin II, catecholamines, endothelin - play an important role in myocardium remodeling; they exert not only vasoactive, but also hypertrophic and profibrotic influence [9-11].

The LV afterload is determined by cardiac output and peripheral vascular resistance. The latter may increase at arteriosclerosis, early development of which is peculiar to patients with CKD.

Significant vascular wall architectonics' alterations were demonstrated both at experimental uremia and in patients with terminal renal failure in the form of fibroelastic intimal thickening and elastic lamella calcification associated with the calcium content increase, extracellular matrix dilation and collagen amount increase at the relative reduction in the content of elastic fibers.

According to the data of G.M. London et al., such a non-atheromatous vascular wall's remodeling disturbs the arterial shock-absorbing function aimed at providing blood flow continuity at the LV diastolic filling due to the formation of the so called repulsion wave in elastic arteries [12]. Forming diastole, it provides return of a part of blood volume that had entered aorta and large arteries, thus providing coronary blood flow continuity in the cardiac cycle's diastolic phase. In case elastic properties weaken, repulsion wave formation time reduces, thus, a part of blood volume that had entered aorta and large arteries during systole earlier than usual, i.e. before the systolic ejection phase finished, reaches ascending aorta; this results in the LV afterload and myocardial oxygen consumption increase. Experimental studies have showed that chronic aortic wall's rigidity directly reduces subendocardial blood flow and cardiac transmural perfusion. The role of arterial shock-absorbing dysfunction in the formation of the LV hypertrophy has been proved many times by studies of G. London et al. 
In particular, these authors demonstrated close connection between pulse wave velocity, which reflects vascular wall's rigidity, and the LV myocardial mass index.

Thus, the LV hypertrophy development at CKD is the result of the increased LV afterload and end diastolic pressure, which is a compensatory mechanism closely associated with mortality of cardiovascular diseases [13]. Much attention has been given to the possibility of the LV hypertrophy regression in patients with $\mathrm{CKD}$ in the setting of effective arterial pressure (AP) control in the last decade. The studies usually involved patients with terminal stage of chronic renal failure, and results of most of them revealed positive influence of hypotensive therapy on the LF myocardium.

Both arterial and myocardium remodeling have a multifactor nature. Some of the factors, such as age, gender and AP are non-specific to chronic renal failure, while volume overload is more specific to uremia.

Thus, vascular wall's remodeling is the adaptive function of its functions and morphology. Functional alterations are connected with vasoconstrictor vascular reactions to transmural pressure and neurohumoral regulation, morphological - with vascular wall's structure alteration [14].

Yu-Lu Liang et al. revealed significant interaction between the common carotid arterial "intima-media" complex (CCA IMC) thickness with the degree of the LF hypertrophy [15]. Other studies revealed the LF hypertrophy in $30 \%$ of children with grade II-IV CKD and in $85 \%$ of patients on dialysis. At the same time, the LF remodeling remained after transplantation of kidneys in 50\% of children [16].

In light of the aforementioned, we may assume that cardiovascular alterations start on early stages in patients with CKD. Adaptive mechanisms peter out quickly; this furthers progression of cardiac and vascular dysfunction. It is extremely important to prevent the development of such complications in patients with CKD in order to improve quality and increase life expectancy in this category of patients.

\section{ENDOTHELIUM AS A REGULATORY ORGAN}

Blood vascular wall consists of 3 concentric layers: intima (internal coat), middle coat and adventitia (external coat). Intima borders with blood vascular lumen and consists of monolayer endothelial cells with minimal amount of lower connective tissue in a normal vessel. Middle coat contains mainly myocytes and elastic fibers (in elastic arteries) and is separated from intima and adventitia by internal and external elastic membranes. Blood vascular endothelial surface functions as a regulatory organ that supports vasomotor tone and 
anti-thrombotic system by regulating adhesion of platelets; it also regulates blood fluidity by discharging anticoagulants and fibrinolytic factors [6, 17].

Endothelial disorder is the initial stage of generalized vascular dysfunction; with the lapse of time, it leads to thickening of internal and external vascular coats, which in the end results in plaque development in large elastic arteries and large and medial muscular arteries. Steady disorder of simple endothelium may lead to endothelial surface instability, fibrous cap's erosion, plaque layering, subclinical plaque tearing and vascular thrombosis. The earliest disorders visible in conorary cardiac vessels and aorta in childhood, including neonatal period, at opening are essentially a fat interlayer, which consists of lipid-filled foam cells and extracellular fat. Fibrous plaques are revealed in 5-10\% of coronary arteries in children of 215 years of age; they initially develop from the fat interlayer [18]. Endothelial dysfunction in children may be caused by such risk factors as young age, coronary diseases in family anamnesis, birth weight and physical activity. Inflammation confirmed by the level of Creactive protein is another important factor of vascular wall's state both in children and adults

[2]. Endothelial function also aggravates in children of 4 years of age with homozygous homocystinuria, although it does not do so in their heterozygous parents. Endothelial dysfunction is also present in children with hereditary hypercholesterolemia. Other conditions with the confirmed endothelial dysfunction in childhood include hereditary conditions concurrent with hyperlipidemia and type I pancreatic diabetes [6, 19-23]. Endothelial dysfunction was also revealed in children with pronounced obesity. CKD usually leads to the development of calcifying arteriopathy in patients, which affect both intima and middle coat of large arteries.

Results of a range of foreign studies indicate that there is an interaction of $\mathrm{AH}$ development risk factors with thickness of carotid arteries; thus, we may assert that endothelial dysfunction is the IMC thickening aspect. Thus, M.B. Lande et al. revealed correlation of IMC thickness with diurnal systolic AP index $(\mathrm{r}=0.57, \mathrm{p}=0.003)$ in children $(\mathrm{n}=28)$ with $\mathrm{AH}$ [24].

\section{“Intima-media” complex thickness evaluation}

Ultrasonic diagnostics allows evaluating wall's structure and vascular lumen and measuring IMC thickness. IMC thickening may be revealing primarily where vessels branch off and blood flow is turbulent [e.g., internal and common carotid arteries near carotid bulb, carotid bulb itself, femoral arteries] [25].

AHA (American Heart Association) data allowed assuming that ultrasonic examination of carotid arteries is one of the methods of evaluating risk of development of complications in 
patients without clinical symptoms, yet belonging to risk group of cardiovascular diseases, including AH. This study may be used in general clinical practice.

A range of studies confirmed efficacy of ultrasonic evaluation of endothelial function (IMC thickness) in order to reveal preclinical vascular diseases and evaluating risk of atherosclerosis and myocardial infarction development in adult patients, regardless of their age. In 2010, P. Costanzo et al. conducted meta-analysis of 41 randomized studies on the basis of the "MEDLINE" and "Cochrane" databases, which had been published before August 2009, in order to check whether there is correlation between CCA IMC thickness and results of the conducted therapy. The studies, which were included in the end analysis, covered 18,307 participants overall of $58 \pm 5$ years of age (43\% of them being women) with cardiovascular diseases of varying etiology, who received therapy (including antihypertensive therapy) aimed at reducing risk of development of cardiovascular complications. According to the results of the conducted study, it was concluded that favorable changes of the vascular wall (i.e., of IMC) in the setting of the conducted therapy do not always reflect improvement of clinical results, despite IMC thickening, which reflects increase in the risk of cardiovascular diseases [26].

In the study of children of patients, who had early myocardial infarction in anamnesis, Cuomo et al. evaluated the possibility of including IMC ultrasonic examination in clinical practice for adolescents with high risk of coronary diseases [27]. However, Sorof et al. were unable to demonstrate correlation between maximal CCA IMC thickness and AP at AH in boys and girls of 6-18 years of age [28]. It is important to note that IMC thickening was observed in young patients, who had had terminal stage of a renal disease in childhood, and in children with terminal stages of CKD. The analysis showed that the thickest CCA IMC was in children on dialysis; however, IMC thickening was also present in recipients with a kidney transplant, and even in children with stage II-IV CKD.

The data of multiple foreign and domestic studies obtained by ultrasonic examination using high-frequency transducers confirm significant correlation of CCA IMC thickness with such endothelial dysfunction development parameters as obesity, hypercholesterolemia, hereditary combined hyperlipidemia, type I pancreatic diabetes both in adults and children [21,29].

According to the data of several studies, CCA IMC thickness in adults depended on age and gender and did not depend on body weight index and physical stress. In contrast with it, the studies of healthy children and adults of 10-25 years of age revealed that carotid arterial IMC thickness does not depend on age and gender in people under 18 years of age. The wall rapidly thickens in males over 18 years of age, so the carotid arterial IMC in boys becomes 
significantly thicker than in girls $(\mathrm{p}<0.001)$. According to the data of other studies, IMC thickness was directly caused by the level of systolic AP and relative body weight [30].

M.B. Lande et al. revealed correlation of CCA IMC with diurnal systolic AP index $(r=0,57$, $\mathrm{p}=0.003)$ in children $(\mathrm{n}=28)$ with AH. Finnish population study OPERA (Oulu Project Elucidating Risk of Atherosclerosis) revealed correlation between the level of systolic AP and CCA IMC. A range of foreign studies of children and adolescents also revealed correlation of AH with endothelial disorders [24, 31].

CCA IMC thickening is described in adults, who had had terminal stage of a renal disease in childhood, and in children with terminal stages of CKD. Thus, the study of carotid arterial IMC in children on dialysis, in recipients with a kidney transplant and children with stage IIIV CKD revealed IMC thickening in all 3 groups; however, it was the most pronounced in patients on dialysis. This study also revealed significant correlation of CCA IMC thickness with AP $(r=0.5, p<0.05)$ [32]. The Kuopio Ischemic Heart Disease Study showed that risk of CCA IMC thickening was 2.61 times higher in patients with $\mathrm{AH}$ than in people with normal AP, while in case systolic AP is higher than $175 \mathrm{~mm} \mathrm{Hg}$, this risk increases 3.61 times. However, Sorof et al. did not reveal correlation between maximal CCA IMC thickness and AP in their study of children of 6-18 years of age with AH [28], while this correlation in children with $\mathrm{AH}$ was revealed in a study by E.M. Urbina et al. [31]. A population study in China revealed CCA IMC thickening in adults patients with terminal CKD. M. Litwin, E. Wuhl et al. confirmed in their study of 56 children with stage II-V CKD that the vascular damage affecting the transversal area of wall and lumen of the carotid artery is especially noticeable in children on dialysis. This study also confirmed quantitative correlation of CCA IMC thickness with AP $(r=0.5, p<0.05)$ [33]. M.I. Yilmaz also revealed correlation of IMC thickness with AP in patients with CKD. At the same time, the study by A. PosadzyMalczynska et al. did not reveal CCA IMC thickness reduction in patients with terminal stage of CKD even after transplantation [32].

Although IMC thickening of common femoral arterial is another parameter of risk of development of cardiovascular complication, only few works of foreign authors are dedicated to IMC thickness studies either in adults or children. A range of authors revealed statistically significant correlations between the LF posterior wall's thickness, ventricular septum and IMC thickness of common femoral arteries. Thus, the study by A. Berni et al. showed direct dependence of IMC thickness of common femoral arteries on the level of AP in adults $(n=865)$ with non-corrected AH [20]. A range of studies also revealed such correlations in adults without clinical manifestations of cardiovascular diseases $(n=1,080)$. In one of the 
foreign studies of adults, IMC thickening of a femoral artery of more than $0.9 \mathrm{~mm}$ was considered to be a reliable factor of increase in the risk cardiovascular complications together with CCA IMC. Evaluation of IMC of femoral arteries in the setting of the conducted antihypertensive therapy in that study allowed revealing slight correlation with the reduction in risk of cardiovascular complications.

We found only 1 foreign work aimed at evaluating IMC of femoral arteries in relatively healthy children, on the basis of which age-specific standards for this parameter were suggested ( $n=247,10-20$ years of age) [18].

Though this issue is undoubtedly urgent, only few foreign works are dedicated to the determination of IMC thickness of carotid and femoral arteries in children and adults with AH.

\section{ENDOTHELIN-1 AS ONE OF THE FACTORS OF ENDOTHELIAL DYSFUNCTION}

Endothelin-1 (Et-1) is a peptide of strong vasoconstrictor action playing an important role in vascular pathology of various genesis, including nephropathies. Studies all around the world revealed that parameters of vascular endothelium (including Et-1 disorders) correlate with the degree of activity and disease severity in patients with chronic glomerulonephritis. The most pronounced alterations revealed were in patients with chronic renal failure; this stimulated further study of endothelial dysfunction at renal affections and cardiovascular pathology development, which is an inseparable part of CKD progression.

Out of all the studied endothelial dysfunction markers only Et-1 was connected with the level of AP in children with nephrotic syndrome; rate of Et-1 content increase increased at stable $\mathrm{AH}$, especially in case of systolic and diastolic AP increase at night. However, W. Wojakowski et al. were unable to reveal significant correlation of the level of blood Et-1 in children with compromised hereditary history of cardiovascular diseases [34].

The data of a range of foreign and domestic studies allow assuming that the level of blood serum Et-1 and of Et-1 urine excretion may reflect the renovascular damage severity. B. Isabelle et al. compared serum Et-1 content in 3 groups of children with chronic renal failure: not on hemodialysis, 24 hours after hemodialysis session and after kidney transplantation. The level of Et-1 in healthy children (from 5 months to 15 years of age) was insignificantly higher than in healthy adults and did not depend on gender and age. In comparison with the control group (healthy children), the level of Et-1 was higher in all the 3 groups; the highest content was observed in children after hemodialysis $(p<0.001)$. That study also revealed negative correlation of the level of blood Et-1 with glomerular filtration rate $(r=-0.39, p<0.01)$ and 
significant correlation of blood serum Et-1 concentration with the osmotic concentration function reduction in the active stag of chronic glomerulonephritis [35]. Given the capability of Et-1 to bind with fibroblasts, thus promoting their spread and extracellular matrix generation, and its chemotactic effect on monocytes (it stimulates synthesis of antiinflammatory cytokines and growth factors), we may assume that it plays a certain role in the development of interstitial fibrosis [36]. Thus, A. Yilmaz that the Et-1 urine excretion level determination (immunosorbent assay) and urine Et-1/creatinine proportion may reflect the degree of development of sclerotic renal alterations (formation of cicatrices) in children, who had had a urinary tract infection [37]. Thus, Et-1 may be one of the tubulointerstitial renal affection markers.

Also, increase in the level of blood serum Et-1 in adult patients with $\mathrm{AH}$ in the setting of diabetic nephropathy was revealed. Positive correlation of the level of blood plasma Et-1 with the degree of atherosclerosis was observed.

\section{CONCLUSION}

Thus, a rather early $\mathrm{AH}$ development in children with renal diseases requires a thorough study of pathogenetic factor, including endothelial dysfunction, and studies of morphological and functional criteria and predictors of disease progression; among them are morphometric heart parameters, IMC thickness alteration in carotid and femoral arteries and the level of blood serum Et-1. Combination of these techniques of studying patients with $\mathrm{AH}$ is not yet widespread, but it deserves a thorough examination.

\section{REFERENCES}

1. National Higt Blood Pressure Education Program Working Group on Higt Blood Pressure in Children and Adolescent. The fourth report on the diagnosis, evaluation and treatment of Higt blood pressure in children and adolescents. Pediatrics. 2004; 114 (2): 555-576.

2. Ridker P.M., Rifai N., Rose L. et al. Comparison of C-reactive protein and low-density lipoprotein cholesterol levels in the prediction of first cardiovascular events. N. Engl. J. Med. 2002; 347: 1557-1565.

3. Alexeeva E.I., Dvoryakovskii I.V., Sugak A.B. CCA wall's structural disorder in children with SLE. Ros. pediatr. zhurn. - Russian pediatric journal. 2010; 3: 36-42.

4. Strogii V.V. Sb. dokl. 4-go s"ezda kardiologov Respubliki Belarus' [Collected Reports of the $4^{\text {th }}$ Congress of Cardiologists of the Republic of Belarus]. Minsk, 2000. pp. 316-317.

5. Kotovskaya E.S., Yur'ev A.S., Parfenova L.M. Possibilities and prospects of evaluating the left ventricular diastolic function at Doppler echocardiography. Kardiologiya - Cardiology. 1997; 10: 66-68.

6. Margieva T.V., Sergeeva T.V., Smirnov I.E. Endothelial dysfunction in chronic glomerulonephritis in children. Vestnik Rossiiskoi akademii meditsinskikh nauk - Annals of the Russian Academy of Medical Sciences. 2008; 12:10. 
7. Chapman J.N., Dobson J., Wilson S. et al. Effect of spironolactone on blood pressure in subjects with resistant hypertension. Hypertension. 2007; 49: 839-845.

8. Zurowska A., Balasz I., Zagozdzon I., Marczak E. Hypertension in children with chronic renal failure. Pol. Merkuriusz. Lek. 2000; 8: 259-260.

9. Badaeva S.V. Myocardial hypertrophy risk factors at CKD. Nefrol. i dializ - Nephrology and dialysis. 2008; 10 (2): 96-101.

10. Yakimenko E.A., Severgina I.A., Polivoda S.N. Experience of the long-term use of fixed lisinopril and amlodipine combination for treating patients with hypertonic disease together with ischemic heart disease. Arter. gipertenz. - Arterial hypertension. 2009; 5 (7): 10-12.

11. Sarnak M.J., Levey A.S., Schoolwerth A.C., Coresh J. et al. Kidney disease as a risk factor for development of cardiovascular disease: a statement from the American Heart Association Councils on Kidney in Cardiovascular Disease, High Blood Pressure Research, Clinical Cardiology, and Epidemiology and Prevention. Hypertension. 2003; 108 (17): 21542169.

12. London G.M., Pannier B., Guerin A.P. et al. Alterations of left ventricular hypertrophy and survival of patients receiving hemodialysis: follow-up of an interventional study. J. Am. Soc. Nephrol. 2001; 12: 2759-2767.

13. Mitsnefes M., Flynn J., Cohn S., Samuels J., Blydt-Hansen T. et al. CKiD Study Group. Masked hypertension associates with left ventricular hypertrophy in children with CKD. $J$. Am. Soc. Nephrol. 2010; 21 (1): 137-144.

14. Berenson G.S., Srinivasan S.R., Bao W. et al. Association between multiple cardiovascular risk factors and atherosclerosis in children and young adults. The Bogalusa Heart Study. N. Engl. J. Med. 1998; 338 (23): 1650-1656.

15. Yu-Lu Liang, Shiel L.M., Teede H., Kotsopoulos D., McNeil J., Cameron J.D., McGrath B.P. Effects of blood pressure, smoking, and their interaction on carotid artery structure and function. Sci. Contrib. Hypertens. 2001; 37: 6-11.

16. Cannella G., Paoletti E., Delfino R. et al. Regression of left ventricular hypertrophy on hypertensive dialyzed uremic patients on long-term antihypertensive therapy. Kidney Int. 1993; 44: 881-886.

17. Biegelsen E.S., Loscalzo J. Endothelial function and atherosclerosis. Coron. Artery Dis. 1999; 10 (4): 241-256.

18. Jurvisalo M.J., Jartti L., Nдntц-Salonen K. et al. Increased aortic intima-media thickness: a marker of preclinical atherosclerosis in high-risk children. Circulation. 2001; 104 (24): 2943-2947.

19. Arnold H. Slyper what vascular ultrasound testing has revealed about pediatric atherogenesis, and a potential clinical role for ultrasound in pediatric risk assessment. J. Clin. Endocr. Metab. 2004; 89 (7): 3089-3095.

20. Berni A., Giuliani A., Tartaglia F. et al. Effect of vascular risk factors on increase in carotid and femoral intima-media thickness. Identification of risk scale. Atherosclerosis. 2011; 216 (1): 109-114.

21. McGill H.C.Jr. Relationship of atherosclerosis in young men to serum lipoprotein cholesterol concentrations and smoking. From the Pathobiological Determinants of Atherosclerosis in Youth (PDAY) Research Group. JAMA. 1990; 264 (23): 3018-3024.

22. Shroff G.R., Sarraf M., Sprenkle M.D., Karim R.M. Air embolism involving the coronary and pulmonary circulation: an unusual cause of sudden cardiac death circulation. Circulation. 2011; 124: 2949-2950.

23. Weissberg P. Mechanisms modifying atherosclerotic disease: from lipids to vascular biology. Atherosclerosis. 1999: 147 (1): 3-10.

24. Lande M.B., Carson N.L., Roy J. et al. Effects of childhood primary hypertension on carotid intima mediathickness. J. Hypertension. 2006; 48 (1): 40-44. 
25. Davis P.H., Dawson J.D., Blecha M.B. et al. Measurement of aortic intima-media thickness in adolescent and young adults. Ultrasound Med. Biol. 2010; 36 (4): 560-565.

26. Costanzo P., Perrone-Filardi P. et al. Does carotid intima-media thickness regression predict reduction of cardiovascular events? J. Am. Coll. Cardiol. 2010; 56 (24): 2006-2020.

27. Cuomo S., Guarini P., Gaeta G. et al. Increase carotid intimamedia thuckness in childrenadolescents, and young adults with a parental history of premature myocardial infarction. Eur. Heart J. 2002; 23 (17): 1345-1350.

28. Sorof J.M., Alexandrov A.V., Cardwell G. et al. Carotid artery intima-media thickness and left ventricular hypertrophy in children with elevated blood pressure. Pediatrics. 2003; 111 : 61-66.

29. Newman III W.P., Wattigney W., Berenson G.S. Autopsy studies in United States children and adolescents. Relationship of risk factors to atherosclerotic lesions. Ann. NY Acad. Sci. 1991; 623: 16-25.

30. Jourdan C., Wuhl E., Litwin M. et al. Normative values for intima-media thickness and distensibility of large artries in healthy adolescents. J. Hypertens. 2005; 23: 1707-1715.

31. Urbina E.M., Khoury P.R., McCoy C. et al. Cardiac and vascular consrquences of prehypertension in youth. J. Clin. Hypertens. (Greenwich). 2011; 13 (5): 332-342.

32. Posadzy-Malaczynska A., Kosch M., Hausberg M. et al. Arterial distensibility, intima media thickness and pulse wave velocity after renal transplantation and in dialysis normotensive patients. Int. Agiol. 2005; 24 (1): 89-94.

33. Litwin M., Wuhl E., Jourdan C. et al. Evolution of large-vessel arteriopathy in pediatric patients with chronic kidney disease. Nephrol. Dial. Transplant. 2008; 23 (8): 2552-2557.

34. Wojakowski W., Gmiski J. Plasma levels of von Willebrand factor, endothelin-1, prostacyclin and thromboxane in children from families with high risk of premature coronary artery disease. Scand. J. Clin. Lab. Invest. 2001; 61 (4): 317-323.

35. Margieva T.V., Sergeeva T.V. Participation markers of endothelial dysfunction in the pathogenesis of chronic glomerulonephritis. Voprosy sovremennoi pediatrii - Current pediatrics. 2006; 5 (3): 22-30.

36. Grzeszczak W., Kowalska K., Kowalski D. Level of endothelin-1 and -2 in plasma of patients with chronic renal failure. Pol. Arch. Med. Wewn. 1994; 91 (2): 84-89.

37. Yilmaz A., Gedikbasi A., Sevketoglu E., Karyagar S., Hatipoglu S., Kiyak A., Mulazimoglu M., Aydogan G., Ozpacaci T. Urine endothelin-1 levels as a predictor of renal scarring in children with urinary tract infections. Clin. Nephrol. 2012; 77 (3): 219-224. 